\title{
Self-mated tribological systems based on multilayer micro/ nanocrystalline CVD diamond coatings
}

\author{
E. Salgueiredo, C.S. Abreu, M. Amaral, F.J. Oliveira, J.R. Gomes, R.F. Silva
}

\begin{abstract}

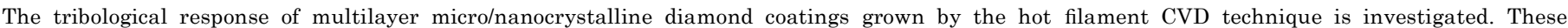

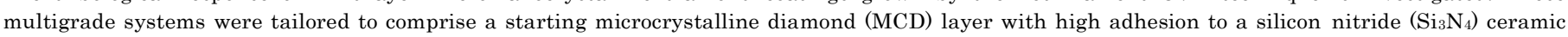

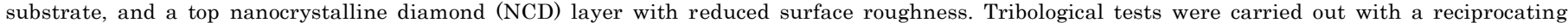

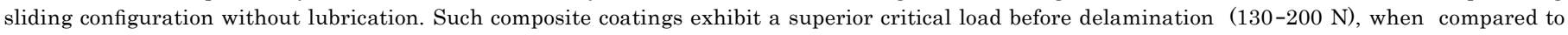
the mono- $(60-100 \mathrm{~N})$ and bilayer coatings $(110 \mathrm{~N})$, considering $\sim 10 \mathrm{~mm}$ thick films. Regarding the friction behaviour, a short-lived initial high friction coefficient was followed by low friction regimes (friction coefficients

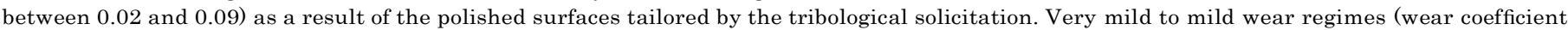

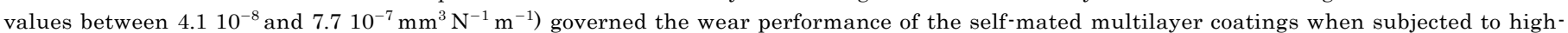
load short-term tests $(60-200 \mathrm{~N} ; 2 \mathrm{~h} ; 86 \mathrm{~m})$ and medium-load endurance tests $(60 \mathrm{~N} ; 16 \mathrm{~h} ; 691 \mathrm{~m})$.
\end{abstract}

\section{Keywords}

Diamond multilayers, Nanocrystalline diamond, Hot filament CVD, Friction, Wear

\section{Introduction}

The intrinsic relatively high surface roughness of microcrystalline diamond (MCD) coatings, when compared to other related films such as the nanocrystalline diamond (NCD) variety or the diamond-like carbon material may hinder its use in several mechanical and tribological potential applications. Such surface characteristic of MCD implies a prevailing mechanical component to the friction phenomenon, resulting from the mechanical interlocking between the micro-sized pyramidal asperities of diamond crystals, which leads to high initial friction values in sliding contacts. This drawback could normally be overcome using NCD films instead, which combine a low surface roughness with the outstanding properties of diamond, namely high hardness, thermal conductivity and chemical inertness [1]. Hence, some of the lowest friction coefficient $(\mu)$ values involving self-mated hard coatings under ambient conditions, without lubrication, were found for NCD ( $\mu \sim 0.01-0.04)$, also offering high wear resistance (wear coefficient $k \sim 10^{-8}-10^{-7} \mathrm{~mm}^{3} \mathrm{~N}^{-1} \mathrm{~m}^{-1}$ ) [2-4]. Nonetheless, these coatings suffered delamination under tribological stress at lower loads $(60 \mathrm{~N})$ compared to those of the MCD system $(80 \mathrm{~N})$ $[5,6]$, due to the higher content of non-diamond species at the grain boundaries and poorer adhesion to the substrate [7].

Thus, multigrade multilayer coatings are here proposed for tribological applications that would combine the higher adhesion of the MCD variety, as the starting layer, with the very low surface roughness of NCD, as the top layer. Particularly, the MCD coatings provide excellent adhesion levels to $\mathrm{Si}_{3} \mathrm{~N}_{4}$ ceramic substrates due to a good thermal expansion match and structural compatibility with diamond [8].

The multilayer coating approach is not unfamiliar in literature, as it is well known to improve the tribological behaviour of conventional systems like ceramic and metallic hard coatings [911], by increasing the adhesion between the coating and the substrate, allowing higher applied loads, surface stress reduction and improving crack propagation resistance [12]. Diamond multilayers were already grown on cemented carbide substrates aiming improvements in the cutting tools performance [13-17]. Sun et al. achieved an upgrade on the working lifetime of drawing dies by a factor of above 15, compared to uncoated ones [16]. Takeuchi and co-workers reported a $30 \%$ increase in the bending strength of the multilayer system, compared to MCD monolayers [13].

Diamond films were also combined in multilayer coatings with other structures, such as nanocubic boron nitride [18], amorphous 
carbon [19] or tetrahedral amorphous carbon (ta-C) [20]. In all cases, the composite structure shows improved properties, when compared to the individual coatings.

The present work compares the tribological behaviour of monolayers, bi- and fourfold multilayers of micro- and nanocrystalline CVD diamond varieties. In all cases, self-mated ball-on-flat systems in dry sliding conditions were tested. To our knowledge, the applied loads used (up to $200 \mathrm{~N}$ ) are considerably higher than those reported in literature.

\section{Experimental}

Dense disc shaped Si N ceramic substrates $(\varnothing 10 \mathrm{~mm} \quad 3 \mathrm{~mm}$ thickness) were ${ }_{\text {pressureless }}$ sintered at $1750{ }_{1}^{\mathrm{C}}$ for $2 \mathrm{~h}$, in a

nitrogen atmosphere. The substrates were then sequentially ground with a $46 \mathrm{~mm}$ diamond wheel, flat lapped with a $15 \mathrm{~mm}$ diamond slurry in an iron/polymer plate. $\mathrm{Si}_{3} \mathrm{~N}_{4}$ commercial balls (Kema Nord) with a $5 \mathrm{~mm}$ diameter were used as counter bodies in the tribological tests. Prior to diamond deposition, both discs and balls were dry etched for 10 min with $\mathrm{CF}_{4}$ in an rf generator (13.56 MHz, EMITECH K1050X) and ultrasonically scratched during $1 \mathrm{~h}$ with a nanometric diamond powder suspension in ethanol, for diamond nucleation enhancement.

An in-house built $3 \mathrm{~kW}$ hot filament reactor was used for chemical vapour deposition of diamond, comprising six tungsten wires $\left(\varnothing_{1} / 9.25 \mathrm{~mm}, 7.5 \mathrm{~mm}\right.$ length). The filaments to substrate distance and the filament temperature were kept constant at about $7 \mathrm{~mm}$ and $\sim 23001 \mathrm{C}$, respectively. A two-colour pyrometer was used to measure the filament temperature. This way, samples were heated at approximately $6301 \mathrm{C}$ by thermal radiation, as measured a K-type thermocouple inserted into the substrate holder, placed on the back side of the substrate. Additional heating of the substrate was achieved by graphite dissipating element fed by a DC external power supply, permitting full temperature control.

Prior to the deposition of the bilayer and multilayer coatings, monolayers of MCD and of two varieties of NCD were deposited using the conditions expressed in Table 1 . In the case of the NCD-1 film, nanocrystals develop as a result of the high methane $\left(\mathrm{CH}_{4}\right)$ concentration (carbon supersaturation) while in the NCD-2 films renucleation is promoted by the partial replacement of hydrogen $\left(\mathrm{H}_{2}\right)$ with argon (Ar) in the gas composition. Then, bilayer and multilayer composites were designed as follows: (i) Bilayer-1: MCD+NCD-1; (ii) Bilayer-2: MCD+NCD-2; (iii) Multilayer-1: MCD $+\mathrm{NCD}-1+\mathrm{MCD}+\mathrm{NCD}-1$ and (iv) Multilayer-2: MCD+NCD-2+MCD + NCD-2. All coatings present a total film thickness in the 10$12 \mathrm{~mm}$ range, for comparison purposes.

Tribological tests of self-mated systems based on diamond coated discs and balls were performed in a ball-on-flat adapted tribometer (PLINT TE67/R) using an unlubricated reciprocating sliding arrangement. All tests were performed in ambient air (R. H. $50 \%-60 \%)$ at room temperature, with constant frequency $(1 \mathrm{~Hz})$ and stroke length $(6 \mathrm{~mm})$. Normal applied loads varied in

Table 1

Hot filament deposition parameters for MCD, NCD-1 and NCD-2.

\begin{tabular}{lllllll} 
Sample & $\begin{array}{l}\text { Deposition } \\
\text { time (h) }\end{array}$ & $\begin{array}{l}\mathrm{CH}_{4} / \mathrm{H}_{2} \\
\text { ratio }\end{array}$ & $\begin{array}{l}\mathrm{Ar} / \mathrm{H}_{2} \\
\text { ratio }\end{array}$ & $\begin{array}{l}\text { Gas flow Total } \\
(\mathrm{sccm})\end{array}$ & $\begin{array}{l}\text { Substrate } \\
\text { pressure } \\
(\mathrm{kPa})\end{array}$ & $\begin{array}{l}\text { temperature } \\
(1 \mathrm{C})\end{array}$ \\
\hline $\mathrm{MCD}$ & 2 & 0.026 & - & 100 & 17.5 & 800 \\
$\mathrm{NCD}-1$ & 3 & 0.073 & - & 100 & 5.0 & 700 \\
NCD-2 & 6 & 0.04 & 0.1 & 200 & 10.0 & 700 \\
& & & & & & \\
\hline
\end{tabular}

the $60-200 \mathrm{~N}$ range. Sliding distances ranged from $86 \mathrm{~m}(2 \mathrm{~h})$ to $\sim 691 \mathrm{~m}$ (16 h, endurance tests) in order to evaluate the short and long term evolution of the tribological response of the monolayers and of the bi- and fourfold diamond multilayers.

Field Emission Scanning Electron Microscopy (FE-SEM) was performed using a Hitachi SU-70 system for surface morphology characterization of the diamond coatings, before and after the tribological tests. UV $\mu$-Raman spectroscopy (HORIBA JOBIN YVON HR800UV), using the line $325 \mathrm{~nm}$ from a He-Cd laser (KIMMON IK series) allowed the identification of the carbon phases. AFM (Nanoscope IIIa, Digital Instruments) using $50 \mathrm{~mm} 50 \mathrm{~mm}$ scans, allowed the determination of the surface roughness of the diamond layers and also of the wear coefficient, using the AFM bearing function for volume loss quantification data [21]. The wear coefficient $(k)$ of the ball specimen was estimated from the nearcircular wear scar dimension, according to the Archard law [22,23]

$$
k=\frac{\pi d^{4}}{64 r W x}
$$

where $d$ is the diameter of the near-circular wear scar, $r$ the ball radius, $W$ the normal applied load and $x$ is the sliding distance.

\section{Results and discussion}

\subsection{As-deposited diamond coatings}

Typical initial root mean square (RMS) surface roughness values for the MCD, NCD-1 and NCD-2 monolayer coatings were 485750, 35071 and $295715 \mathrm{~nm}$, respectively. These AFM measured values are somewhat high, but easily explained by the initial surface roughness of the substrate. The flat lapped surface finishing of the $\mathrm{Si}_{3} \mathrm{~N}_{4}$ substrates presented a RMS value of $\sim 200 \mathrm{~nm}$. Moreover, the 10 min plasma etching with $\mathrm{CF}_{4}$ induced a further increase in surface roughness to $\sim 308 \mathrm{~nm}$. However, plasma etching allows a more effective diamond seeding, and consequently higher adhesion levels of the diamond coating to the substrate [24]. The use of an appropriate surface pre-treatment becomes important to overcome the poor intrinsic adhesion of diamond to most substrates, as a result of its chemical inertness. Another proven advantage of $\mathrm{Si}_{3} \mathrm{~N}_{4}$ ceramics is the similar thermal expansion coefficient to that of diamond, thus diminishing the thermal stress and, therefore, enhancing the coating adhesion $[25,26]$.

Fig. 1 displays cross-sectional SEM images of the as-deposited bilayers ((a), (b)) and multilayers ((c), (d)). As shown in the micrographs, all structures begin with a rough MCD microstructure and end in a smooth top NCD layer. The multilayers present a fourfold configuration, being that Multi-1 and Multi-2 were obtained after doubling Bilayer-1 and Bilayer-2 layers, respectively. The RMS values are $275710,26078,310711$ and $280734 \mathrm{~nm}$ for Bilayer-1, Bilayer-2, Multi-1 and Multi-2, respectively.

\subsection{Worn diamond coatings}

The long term evolution of the tribological response of the diamond coatings was assessed by performing endurance tests with $\sim 691 \mathrm{~m}$ of sliding distance, and a constant applied load of $60 \mathrm{~N}$. This value was chosen based on the lower limit obtained for the critical load tests that were carried out to determine the maximum load prior to film delamination, which are detailed later on.

The worn surfaces of both flat and ball specimens are depicted in Fig. 2. Micrographs in the left column are low magnification views of the wear track regions of Bilayer-1 (a), Multi-1 (c), Bilayer-2 (e) and Multi-2 (g) samples, respectively. Insets depict the corresponding 
a

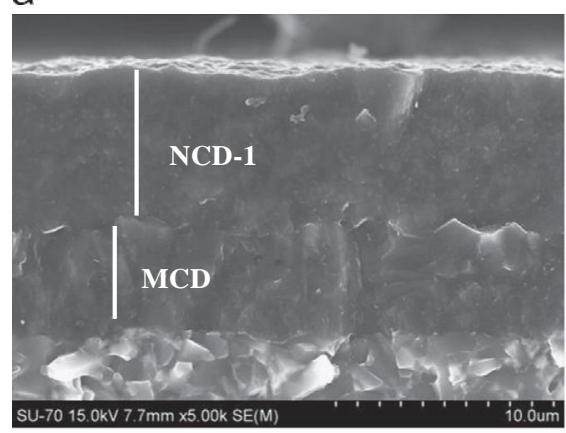

C

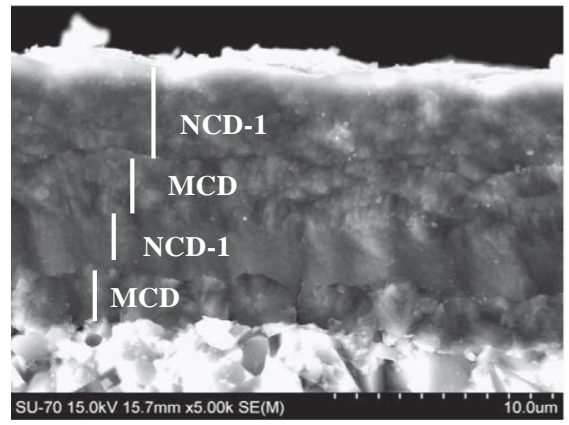

b

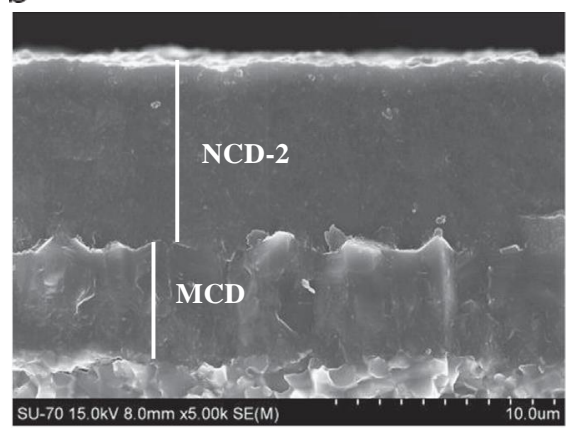

d

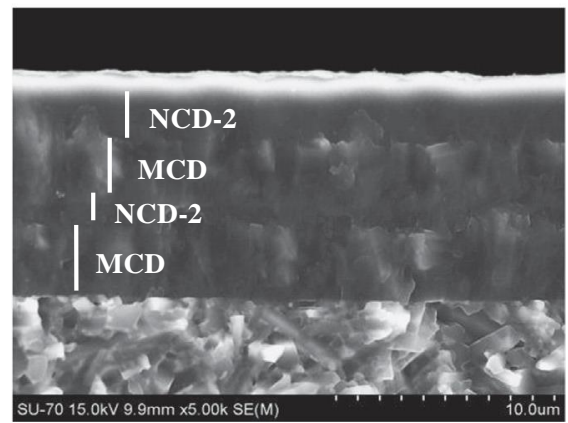

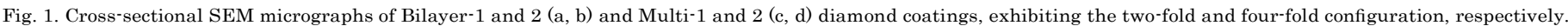

counterpart ball worn surfaces, which were also used to estimate the wear coefficient value according to Eq. (1).

The column in the right side of Fig. 2 shows higher magnification micrographs taken inside the wear track regions. As can be seen, Bilayer- 1 and Multi-1 exhibit smooth polished surfaces (RMS $55738 \mathrm{~nm}$ and $7576 \mathrm{~nm}$, respectively) inside the wear track region, characteristic of a fine-scale abrasive wear mechanism. These endurance tribotests also produced some interspersed cracking leading to some grain pullout. Although the high contact loads considered $(60 \mathrm{~N})$, which equate to applied stresses of 3.5 $\mathrm{GPa}$, the scratch patterns from the substrate polishing were kept inside the wear tracks (Fig. 2(a) and (c)), which evidences the high adhesion level of the diamond coatings. On the other hand, the Bilayer-2 sample shows the presence of several shallow wear grooves (Fig. 2(f)), resulting in higher surface roughness values (RMS $245754 \mathrm{~nm}$ ) than those found on the NCD-1 grade systems. This result can be explained from the higher content of $\mathrm{sp}^{2}$ softer phase present on NCD-2 grade samples, making these systems more prone to abrasion wear damage. In the Multi-2 film, a striking feature is the existence of an angular crack pattern bordering a smooth wear track (RMS $7577 \mathrm{~nm}$ ), superimposed on the aforementioned worn surface characteristics of Bilayer-2. The film cracking could be explained by the collapse of the top NCD-2 layer, where high Hertzian contact stresses, resulting from the non-conformal sphere-on-plane configuration, give rise to their arching. Because the NCD-2 grade systems have similar total thickness, the individual layers on Multi-2 will be thinner than those of Bilayer-2, and as such the top layer of the former will become less resilient to accommodate a similar applied stress, bulging toward the edges of the wear track. It is noteworthy, that the applied load of $60 \mathrm{~N}$ corresponds also to the critical load prior to film detachment assessed for the NCD-2 diamond grade, as reported later on.

In relation to the ball specimens, they are characterised by near-circular wear scars with a smooth polished appearance and the presence of a few pullouts, originating from the brittle fracture of the diamond structures. Moreover, the ball corresponding to the
Bilayer-2 sample evidences the existence of shallow abrasive grooves, like those found on the counterpart plate.

\subsection{Critical load of diamond coatings}

The tribotests were also used to determine the critical load $\left(W_{\mathrm{C}}\right)$ of the diamond coatings prior to film delamination under sliding, which are given in Table 2. Fig. 3 exhibits SEM micrographs of the wear track regions under the estimated critical loads. From top to bottom, the monolayer, bilayer and multilayer films are presented in sequence. Under the drastic loading conditions, a common feature consists in the presence of a mixed pattern of angular and transverse semi-circular cracks typical of an abrasive action from a hard component on a hard surface [27], revealing a micro-cracking abrasive mechanism. As readily seen, the density of micro-cracking on coatings NCD-1 (Fig. 3(a)) and Bilayer-1 (Fig. 3(c)) is significantly higher than the ones found on the corresponding NCD-2 and Bilayer-2 coatings, as a result of the higher brittleness of NCD-1 coming from their higher content of $\mathrm{sp}^{3}$ phase [28]. Comparatively, the NCD-2 ended coatings (Fig. 3(b, d) ) are more prone to grain pullout due to a higher content of $\mathrm{sp}^{2}$ phase. A lower critical load of $60 \mathrm{~N}$ was found for the NCD-2 monolayer when compared to the obtained value for the NCD-1 system $\left(W_{\mathrm{C}}^{1 / 4} 80 \mathrm{~N}\right)$. The high $\mathrm{sp}^{2}$ content at the interface diminishes grain cohesion and bonding strength to the substrate [7], which corroborates the observed behaviour. Bilayer-2 (Fig. 3 (d)), being relatively soft, also evidences abrasion grooves at the wear track border, along the sliding direction.

Higher magnification images are presented on the insets of Fig. 3 to highlight features regarding the tribological behaviour of bilayer and multilayer coatings. The inset in Fig. 3(c), corresponding to Bilayer-1 coating, shows the presence of tribolayers in the lateral regions of the image, resulting from the agglomeration of wear debris particles and their subsequent plastic deformation into adhered layers. In addition, the typical surface morphology of NCD-1 grade can be seen on the tribolayer-free central region of the inset. Showing a distinct behaviour, the Bilayer-2 film 
a

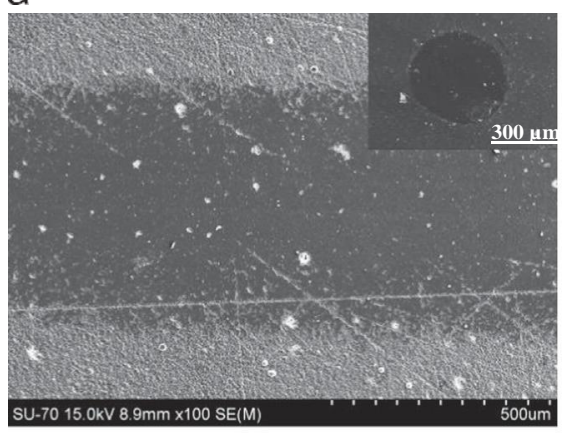

C

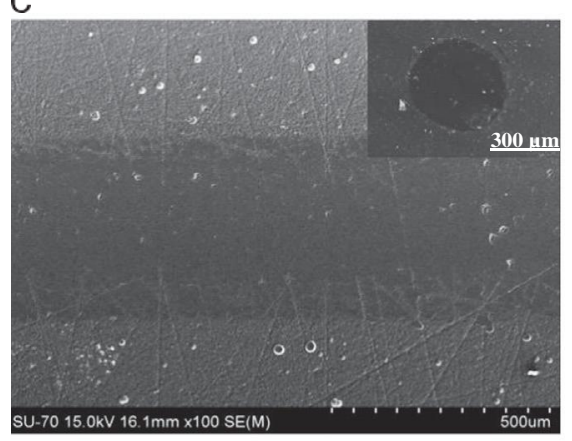

e

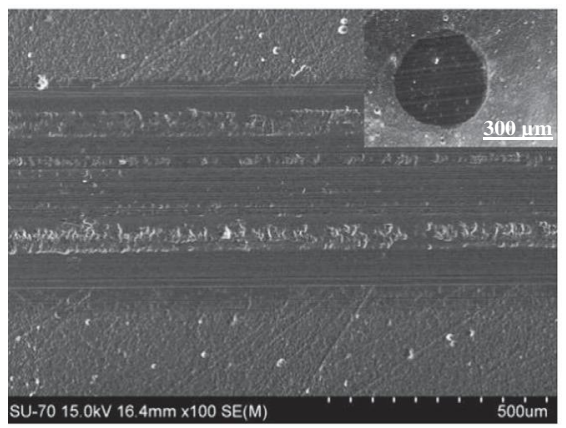

g

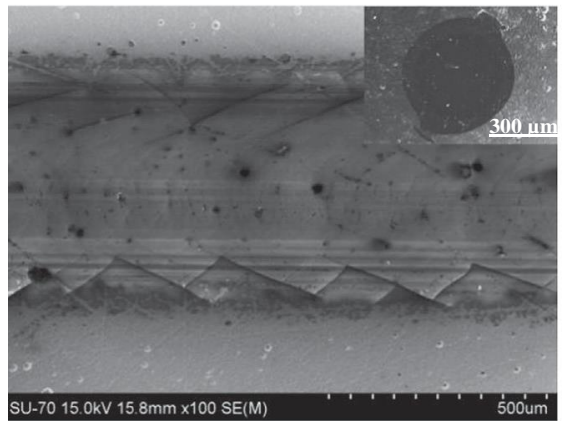

b

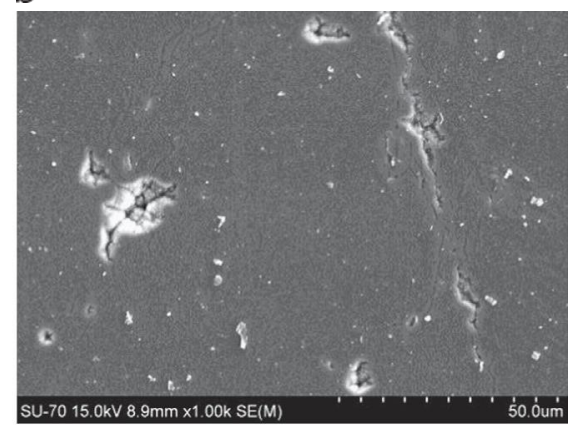

d

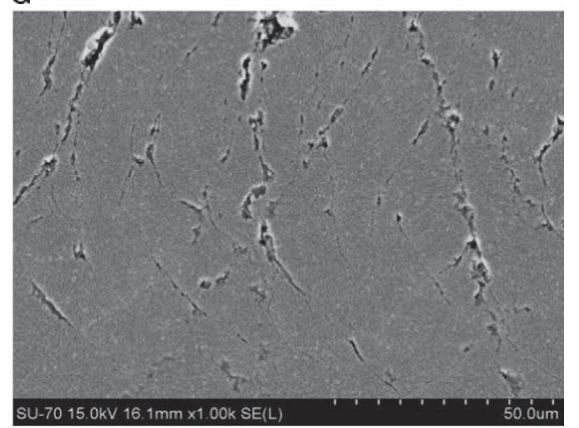

$f$

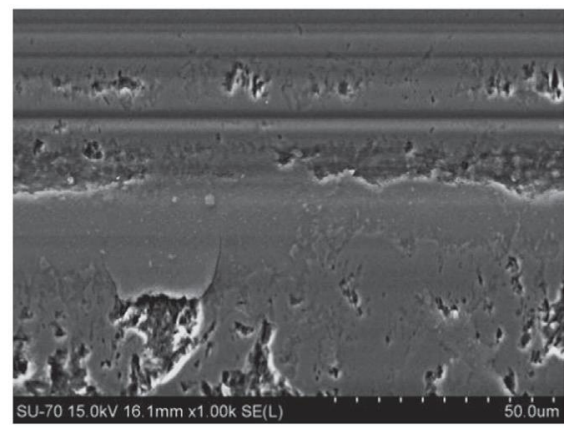

$\mathrm{h}$

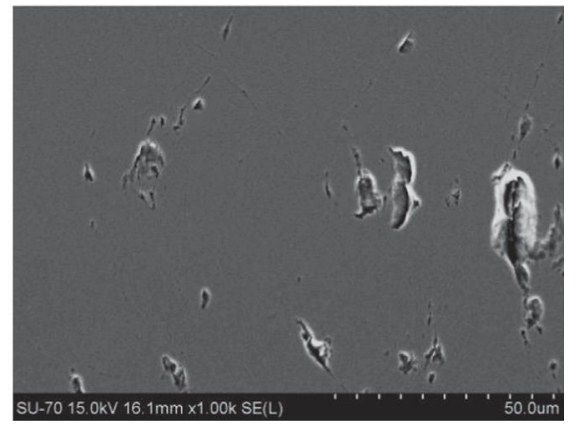

Fig. 2. Morphology of the worn specimens (plate and ball) for Bi-1 (a), Multi-1 (c), Bi-2 (e) and Multi-2 (g) after the endurance tests ( $691 \mathrm{~m})$ at a constant $60 \mathrm{~N}$ load; magnifications inside the wear track for Bi-1, Multi-1, Bi-2 and Multi-2 are presented in images (b), (d), (f) and (h), respectively.

magnification (Fig. 3(d) inset) reveals abraded surface asperities, amid grain pullouts features, without the evidence of tribolayers formation, and parallel grooves typical of abrasion (two-body) by micro-cutting.

Regarding the multilayer systems, both coatings present tribological surfaces with similar features like the presence of grain pullouts, covering the full extent of the wear track. It is noteworthy, that the Multi-2 system sustained a critical load of $200 \mathrm{~N}$, which represents an almost two fold increase when compared to the Multi-1 system ( $130 \mathrm{~N})$, although having similar abrasive wear mechanisms involved. Also, there is a substantial difference in the critical load values of monolayered NCD-2 $(60 \mathrm{~N})$ and its multilayer composite (Multi-2).

Raman spectra of the NCD-2 and Multi-2 films are depicted in Fig. 4(a) and (b), respectively. The shallow penetration depth of the laser radiation used in UV-Raman analysis $\left(\lambda_{1} / 4325 \mathrm{~nm}\right)$ [29] gives a picture of the exposed top layer. In both coatings a sharp diamond peak localized at $\sim 1331 \mathrm{~cm}^{-1}$, combined with the disordered (D) and crystalline (G) graphite bands, at $\sim 1370 \mathrm{~cm}^{-1}$ and $\sim 1580 \mathrm{~cm}^{-1}$, respectively, is visible. The bands located at $\sim 1150 \mathrm{~cm}^{-1}$ and $\sim 1540 \mathrm{~cm}^{-1}$, attributed to intragranular transpolyacetylene (TPA), are commonly accepted as typical of NCD 
films [30]. In the wear track spectra, the shift of the diamond peak outcomes from intrinsic residual stresses associated with the presence of non-diamond material, as well as structural defects, like microtwins, dislocations or impurities [31], created during the sliding action. As can be seen in Fig. 4(a), a positive shift $\Delta v^{1 / 4} 7 \mathrm{~cm}^{-1}$ relative to the value of natural bulk diamond was obtained inside the wear track region of the monolayered NCD-2, which indicates a compressive biaxial stress state of $\sim-4.0 \mathrm{GPa}$,

Table 2

Critical load values, friction coefficient (maximum and average) for the $2 \mathrm{~h}$ at the critical load, and $16 \mathrm{~h}$ at a constant $60 \mathrm{~N}$ load sliding tests of the mono-, bi- and multilayer coatings.

\begin{tabular}{|c|c|c|c|c|c|}
\hline \multirow[t]{2}{*}{ Film } & \multirow[t]{2}{*}{$\mathbf{w}_{\mathrm{c}}$} & \multicolumn{2}{|c|}{$16 \mathrm{~h} @ 60 \mathrm{~N}$} & \multicolumn{2}{|c|}{$2 \mathrm{~h} @ \mathbf{w}_{\mathrm{C}}$} \\
\hline & & $1_{\max }$ & 1 & $1_{\max }$ & 1 \\
\hline MCD & 100 & 0.8 & 0.09 & 0.77 & 0.05 \\
\hline NCD-1 & 80 & 0.75 & 0.06 & 0.78 & 0.03 \\
\hline NCD-2 & 60 & 0.72 & 0.02 & 0.79 & 0.02 \\
\hline Bilayer-1 & 110 & 0.89 & 0.10 & 0.67 & 0.06 \\
\hline Bilayer-2 & 110 & 0.63 & 0.08 & 0.78 & 0.05 \\
\hline Multi-1 & 130 & 0.79 & 0.05 & 0.71 & 0.06 \\
\hline Multi-2 & 200 & 0.75 & 0.09 & 0.87 & 0.06 \\
\hline
\end{tabular}

a

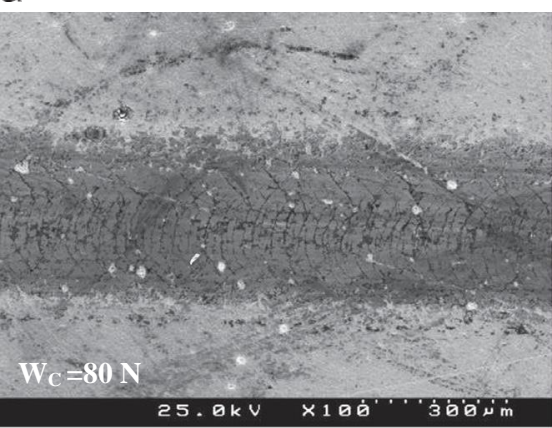

C

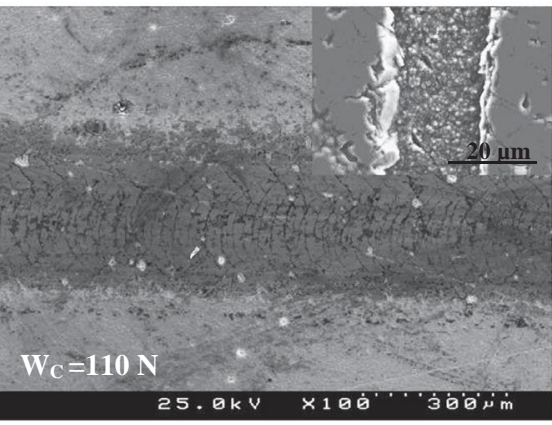

e

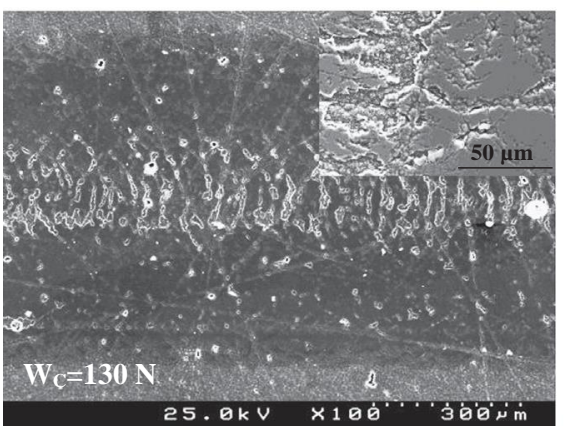

according to Ralchenko's equation [32]. On the other hand, the Multi-2 track reveals a lower compressive biaxial stress of $\sim-2.3 \mathrm{GPa}\left(\Delta \Downarrow_{4} 4 \mathrm{~cm}^{-1}\right)$. Moreover, the Raman spectra acquired inside the same Multi-2 worn track does not present the TPA bands and shows a much less pronounced $G$ band. This indicates that the NCD top layer was almost completely worn out, exposing the underlying MCD layer. The MCD grade is harder and less susceptible to defects creation than NCD, keeping the residual stresses at lower levels. In resume, in the NCD-2 monolayer the tribological solicitation is transposed throughout the coating giving rise to the above mentioned high stress state and consequent film delamination under low applied loads. A great benefit is obtained by the use of the Multi-2 coating where the sacrificial role of the top NCD-2 layer hinders the rise in the residual stress state, allowing its tribological application under much higher loads.

\subsection{Topography of diamond coatings}

A thorough study of the surface topography of the coatings, measured by AFM, was conducted in order to assess the evolution of worn surfaces under sliding and correlate it with prevailing wear damage mechanisms observed by SEM analysis. Depicted in

b

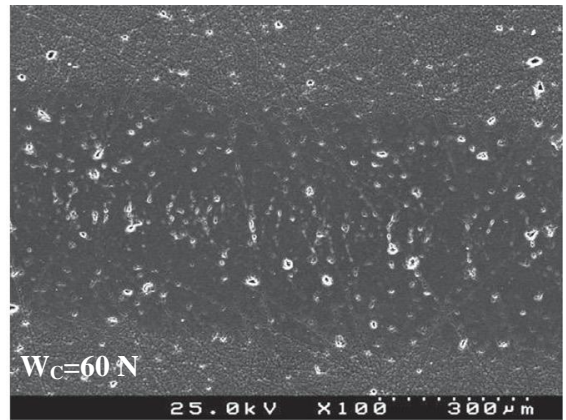

d

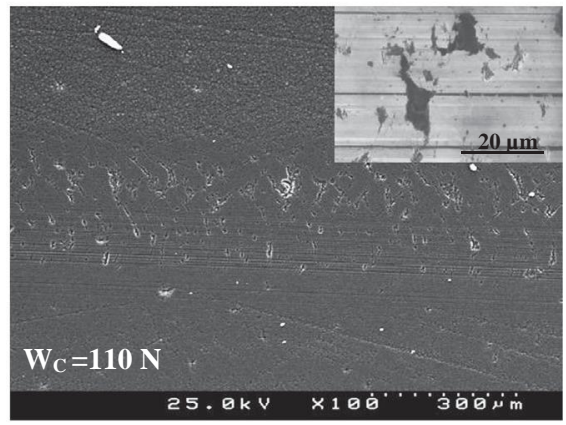

$f$

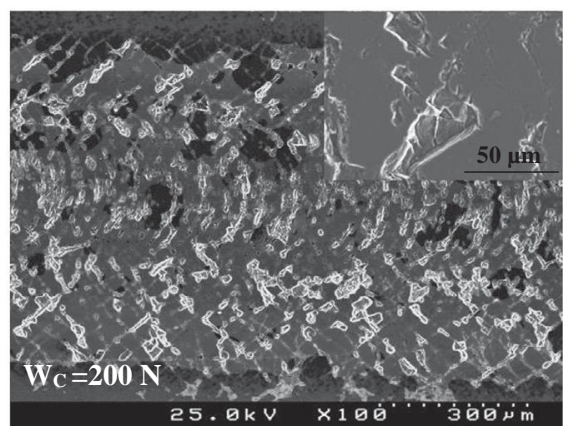

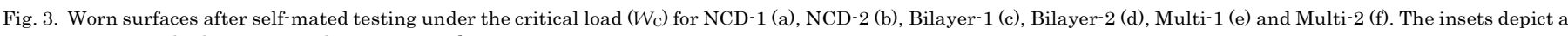
magnification inside the correspondent wear track. 

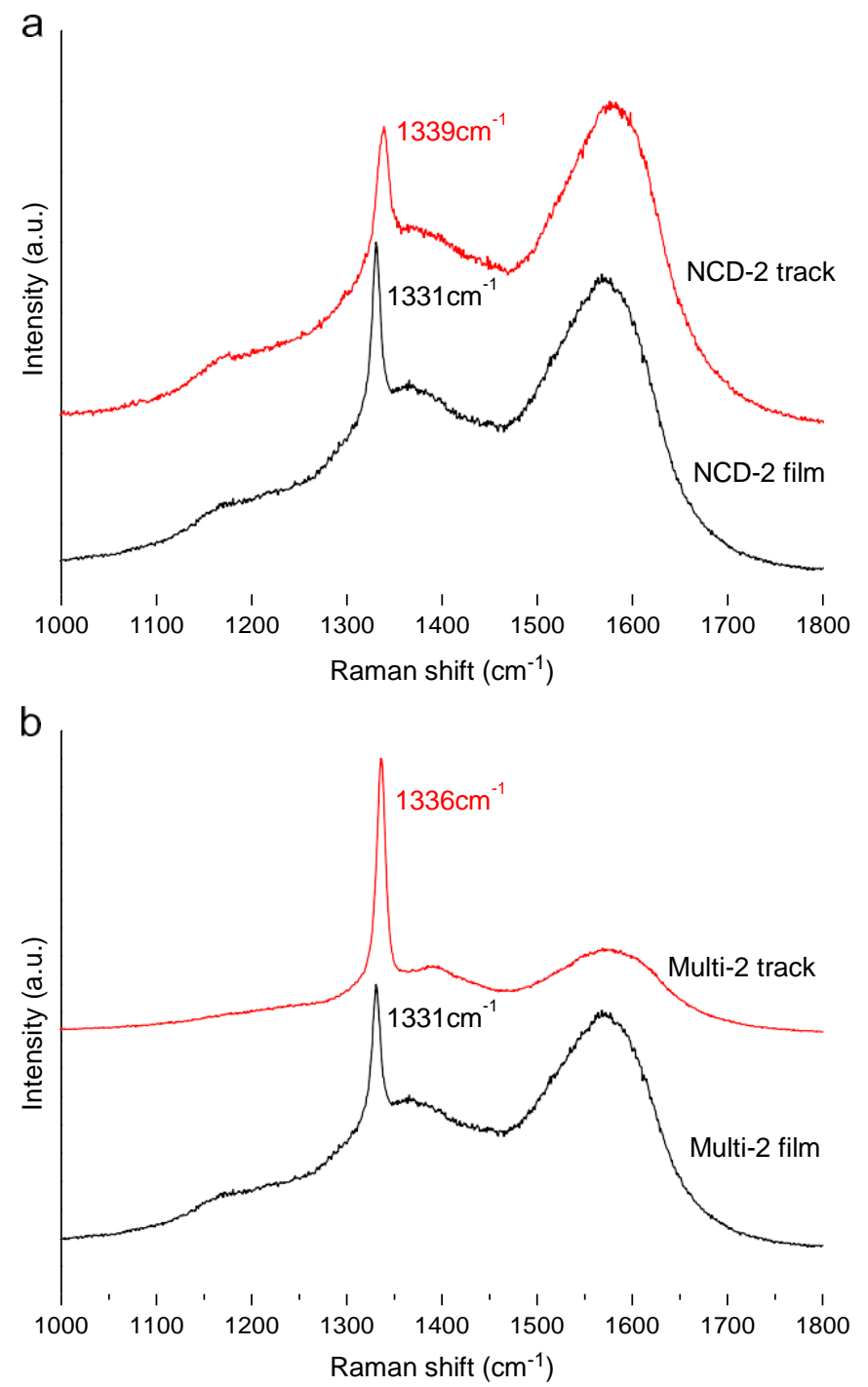

Fig. 4. Raman spectra of the NCD-2 (a) and Multi-2 (b) coatings: unworn film and inside the wear track.

Fig. 5 are AFM scans of the monolayered systems, for as-deposited, endurance tests and worn surfaces under critical load. The asgrown films exhibit topographies which are typical of the involved systems, namely the jagged topography of micropyramidal MCD (RMS $485750 \mathrm{~nm}$ ), a cauliflower shape-like topography of diamond nanoclusters for NCD-2 (RMS $295715 \mathrm{~nm}$ ) and an in between structure for NCD-1, characterized by faceted nanocrystallites (RMS $35071 \mathrm{~nm}$ ). As can be seen, the endurance tests (16 h) are characterized by a complete removal of the aforementioned topography features resulting in very smooth surfaces varying in the range $25-120 \mathrm{~nm}$. These values represent more than a ten-fold decrease in surface roughness for MCD (RMS $4072 \mathrm{~nm}$ ) and NCD-1 (RMS $2572 \mathrm{~nm}$ ), typical of polished surfaces, namely as a result of a micro-cracking abrasive wear mechanism, as above discussed. The NCD-2 coating shows, however, evidences of shallow wear grooves, from micro-cutting abrasive action, leading to the highest surface roughness value (RMS $12075 \mathrm{~nm}$ ).

Although the much higher applied loads, thus the higher severity of contact during the $2 \mathrm{~h}$ critical load tests, the surfaces of MCD and NCD-1 monolayers also present similar abrasive wear mechanisms to those above mentioned. After the initial truncating and progressive blunting of protruding surface asperities, the resulting worn surfaces exhibit some hints of the intrinsic deep valleys of MCD and the depressions of NCD-2 nm-scale roughness. For the later, the initial topography is modified to a lesser extent, as a result of a shorter test under the same applied load $(60 \mathrm{~N})$ as that of the endurance tests. In the case of NCD-1, the initial topography is almost totally abraded as a result of the higher contact load involved $(80 \mathrm{~N})$, comparing to the $\mathrm{NCD}^{-2}$ coating $(60 \mathrm{~N})$.

Regarding the composite coatings, the evolution of their topography under tribological assessment is depicted in Fig. 6. All as-deposited coatings are characterized by the prevalence of elemental structural units spatially organized as diamond nanoclusters, resulting from the nanodiamond top layer. As readily seen, NCD-1 graded systems show a smoother appearance for the worn surfaces subjected to the endurance tribotests. On the other hand, the coatings having a NCD-2 top layer reveal the presence of micro-cutting abrasion grooves, more emphasized on the Bilayer-2 system. The surface finishing condition of NCD-1 systems is coherent with a fine-scale abrasive wear mechanism, as previously reported in the corresponding SEM images (Fig. 2). This characteristic is also supported by the reduction in the surface roughness values of the worn coatings, namely a fivefold decrease for Bilayer1 (RMS $55738 \mathrm{~nm}$ ) and an almost four fold reduction for Multi-1 (RMS $7576 \mathrm{~nm}$ ) and Multi-2 (RMS $8077 \mathrm{~nm}$ ). As for the Bilayer2 coating with a starting roughness of RMS $310711 \mathrm{~nm}$, the decrease in surface roughness is considerably smaller as a result of the formation of deep wear grooves, leading to a final roughness of RMS $245754 \mathrm{~nm}$. Regarding the worn surfaces obtained under the critical load a similar trend is observed. Once more, the Bilayer-2 coating presents a higher surface degradation with a corresponding roughness value of RMS $165738 \mathrm{~nm}$ due to the existence of a dense field of micro-cutting wear grooves. Moreover, the topography of the Multi-1 coating reveals a widespread distribution of grain pullouts as a consequence of the high stresses imposed under the critical load $(130 \mathrm{~N})$. Subjected to the highest measured critical load value $(200 \mathrm{~N})$, the Multi-2 coating is characterized by a smooth topography superimposed on a surface exhibiting some waviness, as a result of the incomplete flattening of wear grooves.

\subsection{Friction and wear coefficients of diamond coatings}

Examples of the friction coefficient evolution of the diamond coatings are depicted in Fig. 7. As can be seen, all curves exhibit a similar trend whereby an initial intense peak, corresponding to static friction values as high as approx. 0.90, is followed by a short running-in regime not exceeding a sliding distance of $10 \mathrm{~m}$. The initial short-lived friction peak results from the intense initial mechanical interlocking between antagonistic hard surface asperities. On the other hand, the subsequently running-in period corresponds to the truncating and subsequent blunting of diamond nanoclusters. Following these two features, a regime of low friction sets in for the remaining length of the tribotests. A summary of representative values for the static friction $\left(\mathrm{m}_{\max }\right)$ and steady-state friction (m) after running-in is presented in Table 2. As expected, based on previous work of this group, the MCD coating is generally characterised by the largest occurring values for the static friction $\left(m_{\max }\right)$ and average values for the friction after running-in (m). This can be explained due to the intrinsic rough starting topography of $\mathrm{MCD}$, which gives rise to a high initial friction response resulting from a more important deformation component in the overall friction phenomenon. The friction behaviour of MCD will serve as a reference material for the other systems, since it constitutes the bottom layer for all composite coatings. The static friction coefficient values are strongly influenced by the surface roughness of all the diamond coatings and, as such, all the studied systems present somewhat 


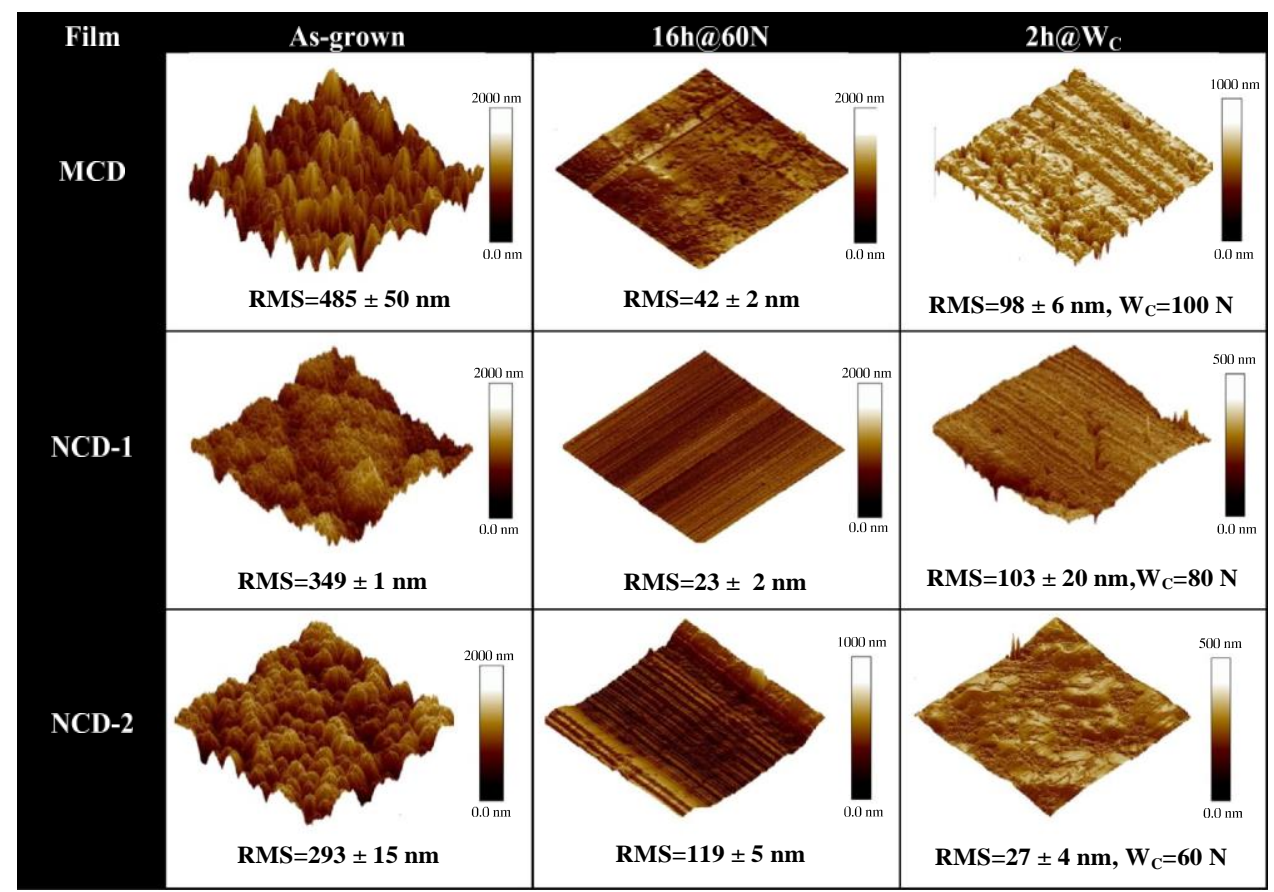

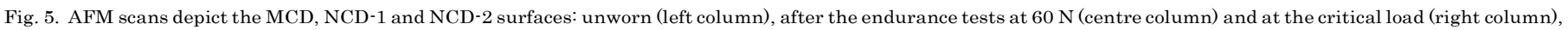
with respective root mean square roughness values.

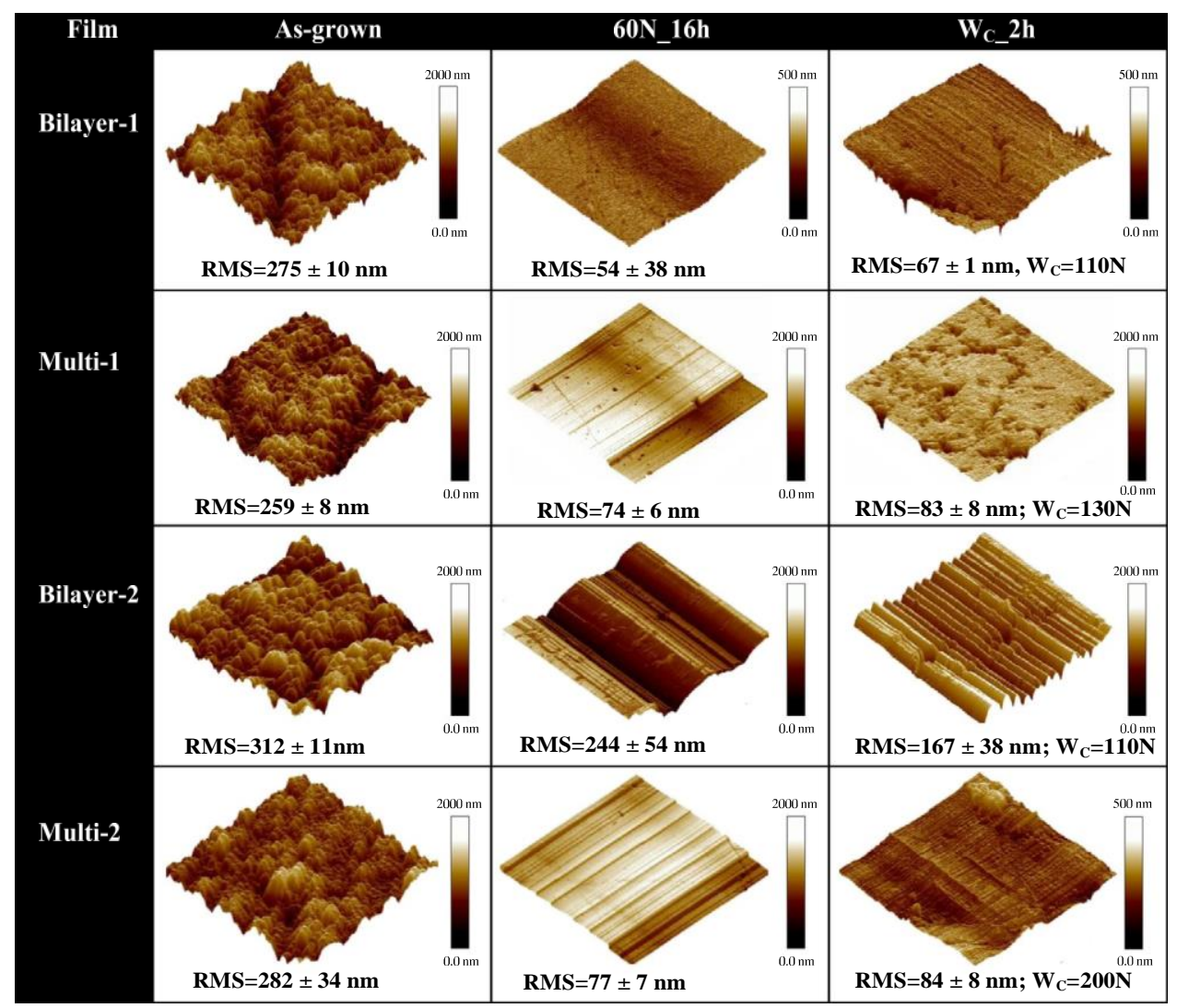

Fig. 6. AFM scans of the as-grown (left column) and worn surfaces after the endurance tests (centre column) and under high loading conditions (right column) for the bilayer and multilayer coatings, with respective root mean square roughness values.

similar values for this feature, exhibiting high $\mathrm{m}_{\max }$ because of the substrate pre-treatment with $\mathrm{CF}_{4}$ plasma. Among, the monolayer coatings, the MCD is characterised by the largest occurring values for $\mathrm{m}$. This can be explained due to the intrinsic rough topography of MCD that is partially kept during the runs. Regarding the monolayered nanocrystalline films, the endurance tribotests $\left(16 \mathrm{~h}, W^{1} / 60 \mathrm{~N}\right)$ were characterised by very low steady-state friction coefficient values, with the NCD-1 coating showing a relatively higher coefficient as a result of the rougher faceted microstructure. On the other hand, the difference in $\mathrm{m}$ for NCD-1 

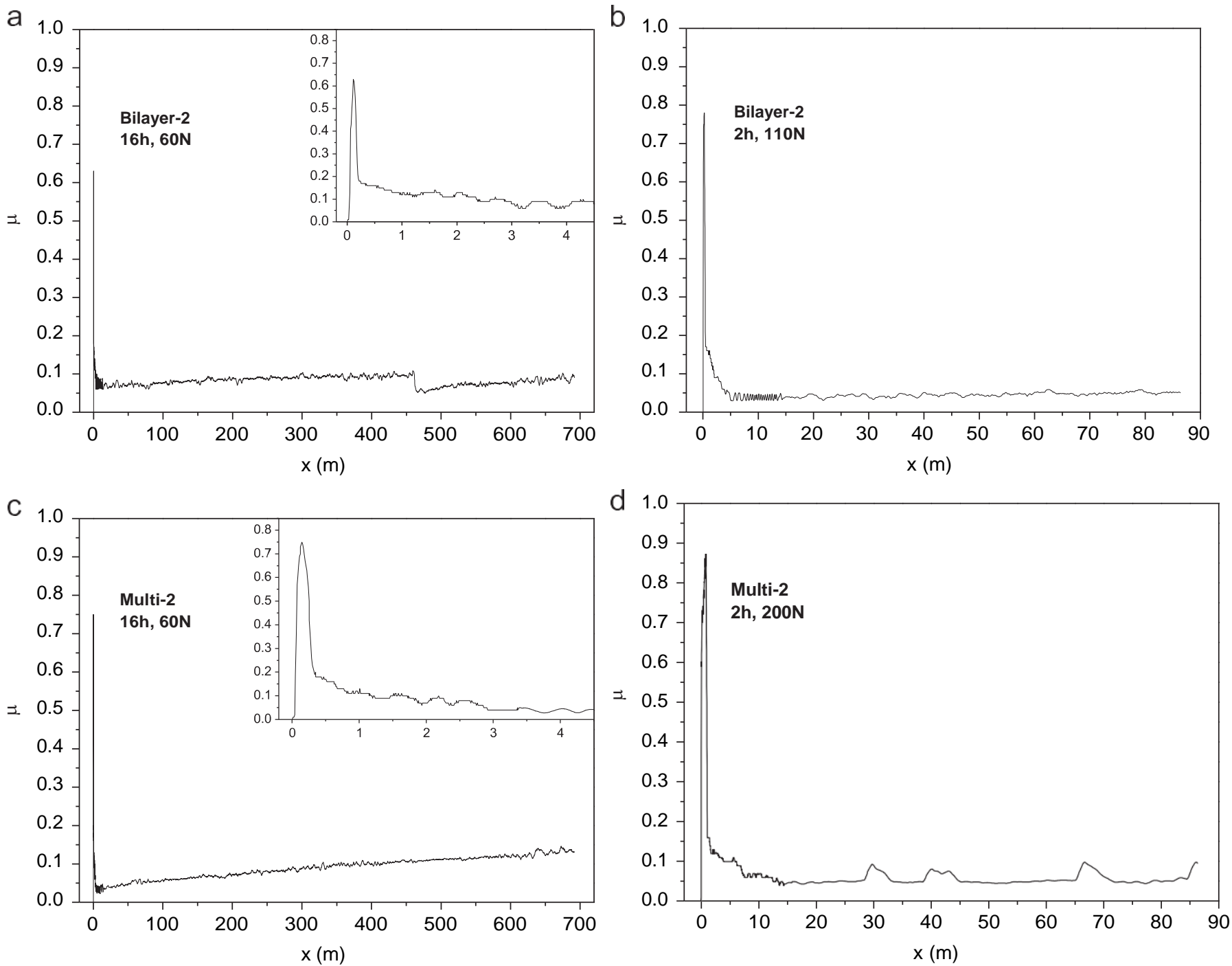

Fig. 7. Friction curve evolution as a function of sliding distance of Bilayer-2 and Multi-2 for the endurance tests ((a), (c)) and critical load tests ((b), (d)). Insets present a detailed view of the narrow peak and running-in period of the endurance tests friction curves.

and NCD-2 when subjected to the critical load is decreased, due to the higher contact stress undergone by the NCD-1 coating. The initial topography is rapidly destroyed leading to a smoothed sliding surface with similar surface features as that of NCD-2, and, therefore, a similar friction interaction.

In Fig. 7, the friction curves of Bilayer-2 and Multi-2 for the endurance tests ((a), (c)) and critical load tests ((b), (d)) are presented side-by-side. A detailed view of the narrow peak and running-in period is depicted on the insets of the endurance tests friction curves. The gradual decrease in friction response corresponds to the accommodation of opposing surfaces and the presence of oscillations to the progressive blunting of nanocluster asperities and wear debris production. Fig. 7(b) shows the friction coefficient of Bilayer-2 subjected to its critical load $(110 \mathrm{~N})$. As readily seen, the initial friction $m_{\max }$ is higher than for the endurance test as a result of the almost two fold increase in contact load, which implies a more intense energy dissipation to initiate the relative motion between surfaces. On the other hand, the low friction regime is characterised by slightly lower average values (0.05) under the critical load, which can be explained once more by the contact pressure increase. In this case, a higher applied load plays a beneficial role by producing smoother surfaces by accelerated blunting of nanodiamond asperities, which can be corroborated by the SEM and AFM data (see Figs. 2, 3 and 6). A similar behaviour is found for the Multi-2 coating. Nonetheless, the endurance curve shows a monotonic increase in $\mathrm{m}$ with the sliding distance. This is due to the progressive wear of the top NCD-2 layer as discussed in Section 3.3. Ultimately, this leads to the influence of the exposed rougher MCD layer and increased friction response. Such phenomenon was also observed for the friction evolution of the Multi-1 coating, to a lesser degree due to the harder and thicker NCD-1 top layer, thus mitigating the influence of the underlying MCD.

Regarding to the wear resistance, all coatings were characterised by mild to very mild wear regimes, the wear coefficients varying in the range of $4.110^{-8}-7.710^{-7} \mathrm{~mm}^{3} \mathrm{~N}^{-1} \mathrm{~m}^{-1}$. The counterpart ball specimens were characterised by wear coefficient values in the leeway $2.5 \quad 10^{-8}-4.4 \quad 10^{-7} \mathrm{~mm}^{3} \mathrm{~N}^{-1} \mathrm{~m}^{-1}$, which were estimated from the wear scars dimensions. As can be seen in Fig. 8(a), corresponding to the endurance experiments $\left(W^{1 / 4} 60 \mathrm{~N}\right)$, all plate specimens were characterised by a lower wear resistance compared to the ball samples, as a result of fatigue effects induced by the intermittent contact during the reciprocating sliding. The Bilayer-2 plate shows the lowest wear resistance (7.7 $10^{-7} \mathrm{~mm}^{3} \mathrm{~N}^{-1} \mathrm{~m}^{-1}$ ) due to the softer nature of the NCD-2 top layer, while the Multi-1 ball exhibited the highest wear resistance 

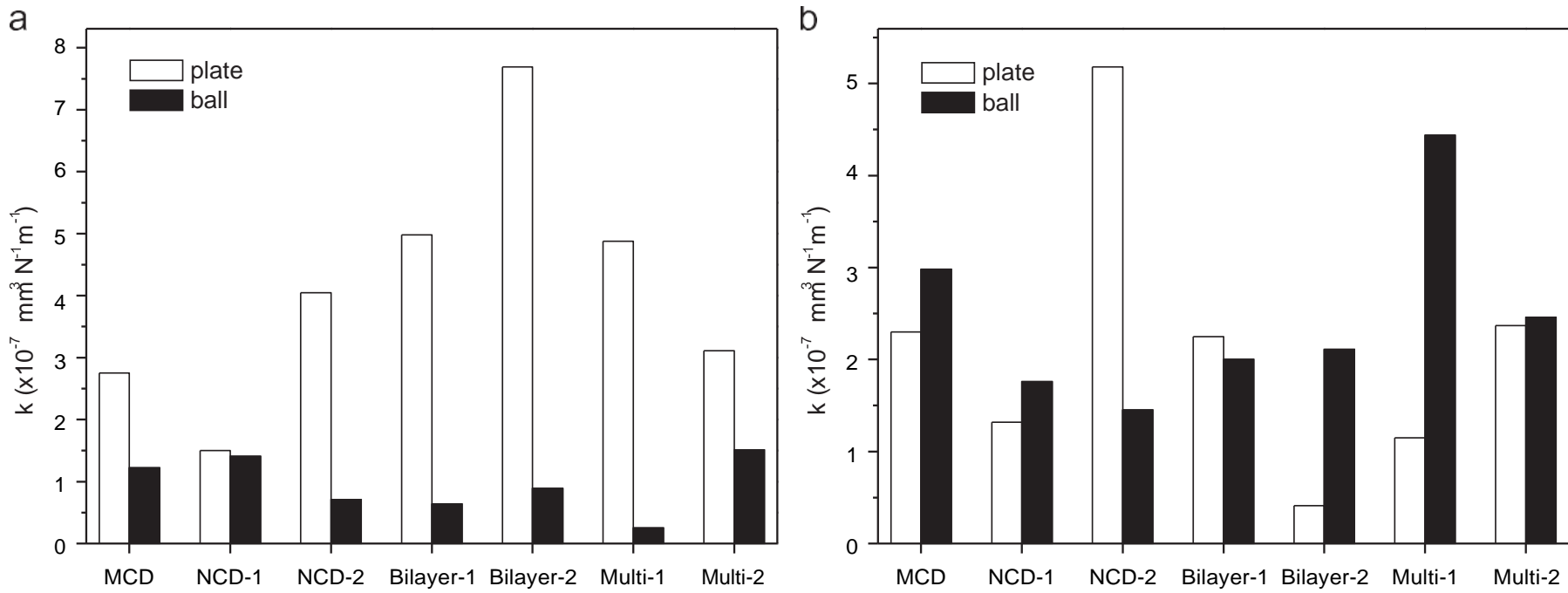

Fig. 8. Wear coefficient values for ball and plate specimens after the endurance experiments (a) and under the critical load, $W_{\mathrm{C}}(\mathrm{b})$, for all diamond systems.

(2.5 $10^{-8} \mathrm{~mm}^{3} \mathrm{~N}^{-1} \mathrm{~m}^{-1}$ ). The later behaviour can be explained by the harder NCD-1 grade top layer combined with the lack of cyclic fatigue effects for the ball specimens.

As depicted in Fig. 8(b), the tribotests under the critical load show a distinct general trend from the endurance experiments. As a rule, the ball specimens show a lower resistance compared to the flat samples. The fatigue phenomenon is still present but the number of cycles is much less than for the endurance tests. Also, the very high contact pressures reached under critical loading will limit the wear life of the ball specimens, since they usually present lower adhesion levels to the substrate when compared to the flat ones [33]. As readily seen, the monolayered NCD-2 plate exhibit the highest wear coefficient due to the increased $\mathrm{sp}^{2}$ content and, as a result, lower shear strength. Nonetheless, NCD-2 graded toplayer composites show an increased wear resistance. This can be explained by the accelerated wearing off of the thin sacrificial NCD-2 top layer, which will expose the underlying MCD coating less prone to subsequent wear damage. Such behaviour can be further corroborated by the similar wear resistances observed in Multi-2 and monolayered MCD systems. It is noteworthy to mention that the plate specimens of Multi-2 were able to perform even under higher applied loads (up to $235 \mathrm{~N}$ ), although without a corresponding wear performance of the ball counterpart due to substrate adhesion limitations. Nevertheless, the coating integrity preservation in both elements of the tribosystem was established as a criterion to validate the critical load of the diamond coatings. Accordingly, of all studied systems the Multi-2 composite exhibited an outstanding threshold load of $200 \mathrm{~N}$ prior to gross film detachment, which highlights the enhanced wear performance under high loads of micro/nanocrystalline diamond multilayer coatings.

\section{Conclusions}

Self-mated tribological systems of monolayer, bilayer (or mono-, bi-.and multilayer) and multilayer micro- and nanocrystalline diamond coatings successfully perform both in high-load $(\max 200 \mathrm{~N})$ short-term tests $(86 \mathrm{~m})$ and endurance tests $(60 \mathrm{~N}$; $691 \mathrm{~m}$ ). Experiments were performed without lubrication, show ${ }^{-}$ ing very low values of the friction coefficient that varied between 0.2 and 0.09. Also, very mild to mild wear regimes (wear coefficient values between $4.1 \quad 10^{-8}$ and $7.7 \quad 10^{-7} \mathrm{~mm}^{3} \mathrm{~N}^{-1} \mathrm{~m}^{-1}$ ) were attained.
The fourfold diamond multilayers, characterised by a top most nanocrystalline diamond layer containing a higher amount of $\mathrm{sp}^{2}$ carbon, outperform the other varieties of diamond coatings by withstanding an applied load value of $200 \mathrm{~N}$ without delamination, combined with a high wear resistance $\left(\sim 2.4 \quad 10^{-7} \mathrm{~mm}^{3} \mathrm{~N}^{-1} \mathrm{~m}^{-1}\right)$ and a low friction coefficient (0.06). In this composite coating, the top nanocrystalline diamond layer progressively wears out performing a sacrificial role, while the underneath harder microcrystalline diamond layer keeps the residual stresses at lower levels. Such superior performance validates the great potential offered by these systems in highly demanding tribological applications subjected to high contact pressures where lubrication is not practical, or in applications needing low friction coefficients combined with high wear resistance.

\section{Acknowledgements}

This work was funded by Projects MULTIDIACOAT - PTDC/EMETME/100689/2008 and Pest-C/CTM/LA0011/2011 from Fundação para a Ciência e Tecnologia (FCT, Portugal). E. Salgueiredo and M. Amaral also acknowledge FCT for the Grants SFRH/BD/41757/2007 and SFRH/BPD/26787/2006, respectively. A.J.S. Fernandes from Physics Department of Aveiro University is gratefully acknowledged for the Raman spectroscopy analysis.

\section{References}

[1] O.A. Williams, Diamond and Related Materials 20 (2011) 621-640.

[2] C.S. Abreu, M. Amaral, A.J.S. Fernandes, F.J. Oliveira, R.F. Silva, J.R. Gomes, Diamond and Related Materials 15 (2006) 739-744.

[3] C.S. Abreu, M. Amaral, A.J.S. Fernandes, F.J. Oliveira, J.R. Gomes, R.F. Silva, Diamond and Related Materials 15 (2006) 2024-2028.

[4] C.S. Abreu, M. Amaral, F.J. Oliveira, A. Tallaire, F. Bénédic, O. Syll, G. Cicala, J. R. Gomes, R.F. Silva, Surface and Coatings Technology 200 (2006) 6235-6239.

[5] C.S. Abreu, F.J. Oliveira, M. Belmonte, A.J.S. Fernandes, J.R. Gomes, R.F. Silva, Tribology Letters 21 (2006) 141-151.

[6] C.S. Abreu, F.J. Oliveira, M. Belmonte, A.J.S. Fernandes, R.F. Silva, J.R. Gomes, Wear 259 (2005) 771-778.

[7] K. Mallika, R. Komanduri, Thin Solid Films 396 (2001) 146-166.

[8] M.R. Soares, M. Belmonte, R.F. Silva, Journal of Applied Physics 94 (2003) 5633-5638.

[9] A.A. Voevodin, J.S. Zabinski, C. Muratore, Tsinghua Science and Technology 10 (2005) 665-679.

[10] J. Romero, A. Lousa, E. Martínez, J. Esteve, Surface and Coatings Technology 163$164 \quad$ (2003) 392-397.

[11] Y.M. Zhou, R. Asaki, K. Higashi, W.H. Soe, R. Yamamoto, Surface and Coatings Technology 130 (2000) 9-14.

[12] K. Holmberg, H. Ronkainen, A. Matthews, Ceramics International 26 (2000) 787-795. 
[13] S. Takeuchi, M. Kojima, S. Takano, K. Kazutaka, M. Murakawa, Thin Solid Films 469-470 (2004) 190-193.

[14] L. Schafer, M. Höfer, R. Kröger, Thin Solid Films 515 (2006) 1017-1024.

[15] M. Vojs, M. Veselý, R. Redhammer, J. Janík, M. Kadlečíková, T. Daniš, M. Marton, M. Michalka, P. Šutta, Diamond and Related Materials 14 (2005) 613-616.

[16] F. Sun, Y. Ma, B. Shen, Z. Zhang, M. Chen, Diamond and Related Materials 18 (2009) 276-282.

[17] M. Kadlečíková, M. Vojs, J. Breza, M. Veselý, Z. Frgala, M. Michalka, J. Matějková, A. Vojačková, T. Daniš, M. Marton, Microelectronics Journal 38 (2007) 20-23.

[18] H.Q. Li, K.M. Leung, K.L. Ma, Q. Ye, Y.M. Chong, Y.S. Zou, W.J. Zhang, S.T. Lee, . Bello, Applied Physics Letters 91 (2007) 201918

[19] C. Popov, W. Kulisch, M. Jelinek, A. Bock, J. Strnad, Thin Solid Films 494 (2006) 92-97.

[20] X. Chen, Z. Peng, X. Yu, Z. Fu, W. Yue, C. Wang, Applied Surface Science 257 (2011) 3180-3186.

[21] M. Amaral, C.S. Abreu, F.J. Oliveira, J.R. Gomes, R.F. Silva, Diamond and Related Materials 16 (2007) 790-795.

[22] K.L. Rutherford, I.M. Hutchings, Surface and Coatings Technology 79 (1996) 231-239.

23]
I.M. Hutchings, Tribology-Friction and Wear of Engineering Materials, Butterworth-Heinmann, UK, 2003.

[24] M. Belmonte, V.A. Silva, A.J.S. Fernandes, F. Costa, R.F. Silva, Journal of the American Ceramic Society86 (5) (2003) 749-754.

[25] S.J. Bull, Diamond and Related Materials 4 (1995) 827-836.

[26] M. Belmonte, A.J.S. Fernandes, F.M. Costa, F.J. Oliveira, R.F. Silva, Diamond and Related Materials 12 (2003) 733-737.

[27] K. Holmberg, A. Laukkanen, H. Ronkainen, K. Wallin, S. Varjus, Wear 254 (2003) 278-291.

[28] F.A. Almeida, M. Amaral, F.J. Oliveira, A.J.S. Fernandes, R.F. Silva, Vacuum 81 (2007) $1443-1447$

[29] S. Gupta, B.R. Weiner, W.H. Nelson, G. Morell, Journal of Raman Spectroscopy 34 (2003) 192-198.

[30] A.C. Ferrari, J. Robertson, Physical Review B 63 (2001)121405-4.

[31] N.G. Ferreira, E. Abramof, N.F. Leite, E.J. Corat, V.J. Trava-Airoldi, Journal of Applied Physics 91 (2002) 2466-2472.

[32] V.G. Ralchenko, A.A. Smolin, V.G. Pereverzev, E.D. Obraztsova, K G. Korotoushenko, V.I. Konov, Y.V. Lakhotkin, E.N. Loubnin, Diamond and Related Materials 4 (1995) 754-758.

[33] M.J. Papo, S.A. Catledge, C. Machado, S. Kashef, A.E. Eberhardt, Y.K. Vohra, Materials Research Society Symposium Proceedings 791 (2004) 271-276. 